\title{
Prevalencia de la infección por Chlamydia trachomatis y su potencial impacto perinatal en pacientes chilenas
}

\author{
Prevalence of Chlamydia trachomatis infection and a potential perinatal impact \\ in Chilean patients
}

Paulina Velasquez N. ${ }^{1}$, Priscilla Brebi M. ${ }^{2}$ y Fernando Abarzúa C. ${ }^{3}$

\begin{abstract}
${ }^{1}$ Interna de Medicina, Universidad de La Frontera, Temuco, Región de La Araucanía, Chile.

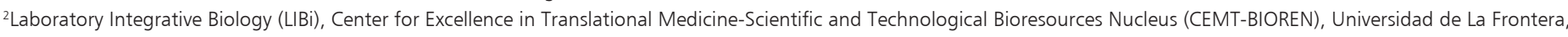
Temuco, Chile.
\end{abstract}

${ }_{3}^{3}$ Servicio Medicina Materno Fetal Hospital Regional Hernán Henríquez y Clínica Alemana Temuco, Región de La Araucanía, Chile.

Sin conflictos de interés.

Financiamiento: no hubo.

Recibido: 16 de enero de 2021 / Aceptado: 5 de julio de 2021

\section{Resumen}

Chlamydia trachomatis es la infección de transmisión sexual bacteriana más frecuente en el mundo. Según datos de la Organización Mundial de la Salud, su prevalencia se estima alrededor de $4,2 \%$ en mujeres. Es una infección silente; sin embargo, puede desarrollar complicaciones en la fertilidad o durante el embarazo. El objetivo de esta revisión es describir la prevalencia de C. trachomatis en estudios recientes en Chile, que utilicen para su detección reacción de polimerasa en cadena (RPC), revisar las posibles complicaciones perinatales asociadas, conocer las recomendaciones de tamizaje en gestantes en otros países y discutir la necesidad de incluir en nuestro país un programa de tamizaje prenatal.

Palabras clave: Chlamydia trachomatis; cribado; complicaciones perinatales; ITS; parto prematuro.

\section{Introducción}

$\mathrm{L}$ a infección genital por Chlamydia trachomatis corresponde a la enfermedad de transmisión sexual bacteriana más frecuente en el mundo. En el año 2012, la Organización Mundial de la Salud (OMS) estimó una incidencia anual, en personas entre 14-49 años, de alrededor de 131 millones de $\operatorname{casos}^{1}$. En el 2018, los Centros de Control de Enfermedades y Prevención, (CDC por su sigla en inglés),

\begin{abstract}
Chlamydia trachomatis is the most frequent bacterial sexually transmitted disease around the world. Estimated prevalence by WHO is $4,2 \%$ for women. Most cases are asymptomatic, but complications in fertility and during pregnancy are possible. The aim of this review is to describe the prevalence of $C$. trachomatis in Chilean studies using polymerase chain reaction (PCR) for detection, to describe the possible perinatal complications, to know recommendations about pregnancy screening in other countries, and to discuss the possibility of implementing in Chile.

Keywords: Chlamydia trachomatis; screening; perinatal complications; STD, preterm delivery.
\end{abstract}

de Atlanta, Estados Unidos de América (E.U.A.), reportó alrededor de 1,8 millones de casos, de los cuales $44 \%$ corresponden a mujeres, con mayor prevalencia en jóvenes entre 15-24 años ${ }^{2-4}$. La OMS estima una prevalencia general de $4,2 \%$ en mujeres y $2,7 \%$ en hombres ${ }^{1}$. En Chile, en estudios que se realizaron en la Región Metropolitana antes del 2015, se describió una prevalencia entre 55 y 79 por 1.000 mujeres de 15 a 24 años ${ }^{5}$.

Chlamydia trachomatis es una bacteria gramnegativa intracelular 
estricta, que tiene la capacidad de fijarse a células epiteliales cilíndricas o de transición del aparato genital, respiratorio o epitelio conjuntival. La infección genera una respuesta inmunológica que puede durar meses o años si no es tratada ${ }^{6}$. El espectro clínico en mujeres incluye uretritis, cervicitis y proceso inflamatorio pélvico. Este último es la principal causa de infertilidad en el mundo y también es causa de dolor pélvico crónico y embarazo ectópico ${ }^{7}$. Se estima que aproximadamente $80 \%$ de las mujeres infectadas son asintomáticas en el momento de la adquisición y pueden desarrollar complicaciones y secuelas de largo plazo ${ }^{8}$.

La infección materna del canal del parto puede provocar complicaciones en el neonato como neumonía y conjuntivitis $^{6,9}$. Además, distintas investigaciones han estudiado la asociación de la infección urogenital con complicaciones obstétricas como aborto espontáneo, parto prematuro, bajo peso al nacer, restricción del crecimiento fetal, rotura prematura de membranas, mortalidad perinatal y endometritis, entre otras; sin embargo, hay diferencias en cuanto a la asociación reportada por distintos autores y la calidad de estos estudios, como se describirá más adelante ${ }^{10,11}$.

La principal forma de contagio es la vía sexual, ya sea vaginal, oral o anal sin protección, siendo el semen un posible transportador de distintos agentes infecciosos, entre ellos C. trachomatis, capaz de adherirse a la cabeza o flagelo del espermatozoide ${ }^{12}$.

Como método diagnóstico las técnicas de amplificación de ácidos nucleicos (NAAT, de la sigla en inglés) son el estándar de oro, dada su alta sensibilidad de 93,8\% y especificidad de $100 \%{ }^{13}$, dejando obsoletos los cultivos, técnicas de inmunofluorescencia y serología. Dentro de las NAAT, la técnica más usada es la reacción de polimerasa en cadena (RPC), que permite el diagnóstico desde muestras de orina, región vulvovaginal, uretra, recto, cérvix, faringe y epitelio conjuntival, teniendo resultados similares en muestras de orina y endocervicales ${ }^{1,14}$.

Para la obtención de muestras endocervicales está validado el uso de cepillado de cuello uterino (citobrush). Además, existen métodos de autotoma de secreción vaginal y orina de primer chorro, convirtiéndose este último en un método de recolección económico, poco invasivo y cómodo para los pacientes, que permite el muestreo en hombres y mujeres ${ }^{13}$. Éstos son utilizados en países con programas de cribado en atención primaria, y en la misma línea se han desarrollado kits de RPC múltiple para detectar más de una infección en una sola muestra, por ejemplo, aquellos que permiten evaluar infección por C. trachomatis y Neisseria gonorrhoeae simultáneamente ${ }^{1,13,14}$. En el caso de mujeres, dado que la infección puede producir cervicitis y uretritis, tanto sincrónica como asincrónicamente, se sugiere complementar el estudio de muestra de orina con muestra endocervical ${ }^{13}$.
Distintos países han implementado programas de cribado en grupos de mayor prevalencia para permitir el diagnóstico y tratamiento oportuno, y de esta manera, disminuir la transmisión y las complicaciones mencionadas.

El objetivo de esta revisión es evaluar la prevalencia de C. trachomatis en Chile, de acuerdo a estudios recientes que utilizan RPC para su detección, revisar el impacto perinatal de la infección en mujeres embarazadas, conocer las recomendaciones de cribado en embarazo en otros países y discutir la necesidad de incluir en nuestro país un programa de cribado prenatal.

\section{Estudios de prevalencia en Chile}

En Chile, la infección por C. trachomatis no es una enfermedad de notificación obligatoria; por lo tanto, no existen reportes ministeriales sobre la prevalencia de la infección en población general ni en mujeres embarazadas. Los datos disponibles provienen de estudios realizados principalmente en la Región Metropolitana, en pacientes de estratos socioeconómicos medios y altos, dado que los test de RPC están principalmente disponibles en centros privados, y pocos incluyen mujeres embarazadas. En general, estudios nacionales e internacionales sugieren que la prevalencia depende del nivel socioeconómico y se asocia a los factores de riesgo descritos, sobre todo en población joven 5 .

En un estudio reciente publicado el año 2018, Huneeus y cols. ${ }^{15}$, evaluaron la prevalencia de C. trachomatis y $N$. gonorrhoeae en adolescentes y adultos jóvenes sexualmente activos, voluntarios, menores de 24 años. Del total seleccionado (286 pacientes), 171 eran mujeres, de las cuales $82 \%$ fueron clasificadas como estrato socioeconómico alto. Las muestras fueron autotoma vaginal y se aplicó una encuesta que incluía datos demográficos, educacionales, presencia de síntomas y conducta sexual. Las muestras fueron analizadas con APTIMA $2^{\circledR}$, que permite la evaluación simultánea de ambas infecciones. Se describe una prevalencia de $C$ trachomatis de 8,8\% en mujeres y $8,7 \%$ en hombres. Sólo $15,9 \%$ declaró usar siempre condón y de ellos, ninguno resultó positivo para C. trachomatis. Por otra parte, tener educación universitaria se describe como un factor protector (OR: 0,28, IC 0,09-0,89) y tener un mayor número de parejas sexuales un factor de riesgo (OR: 1,19; IC 1,1-1,3). Respecto a los síntomas se describe una asociación entre el sangrado post coital y la infección (OR: 4;6; IC 1,3-16,2). El mismo grupo había reportado en $2009^{16}$ una tasa de $6,9 \%$ en mujeres no embarazadas y 19\% en embarazadas (Tabla 1).

En otro estudio similar, Zamboni y cols. ${ }^{5}$, evaluaron la prevalencia de la infección en población femenina sexualmente activa, entre 15 y 24 años, quienes asistieron a un control ginecológico de rutina en tres centros de la Región Metropolitana. Se incluyeron 181 mujeres, 


\begin{tabular}{|c|c|c|c|c|c|c|c|}
\hline Autor & Año & Región & $\mathbf{N}$ & Método diagnóstico & Grupo & Embarazo & Prevalencia \\
\hline Riquelme* & 2019 & La Araucanía & 804 & $\mathrm{RPC}$ & 18-65 años & No & $8,1 \%$ \\
\hline Huneeus $^{15}$ & 2018 & RM & 171 & RPC: muestra genital & $<24$ años & No & $8,8 \%$ \\
\hline Palma ${ }^{20}$ & 2018 & RM & 85 & RPC: muestra fondo de saco vaginal & Mujeres embarazadas & Sí & $12,9 \%$ \\
\hline Zamboni ${ }^{5}$ & 2016 & $\mathrm{RM}$ & 181 & RPC muestra endocervical & $15-25$ años & Sí (8) & $5,5 \%$ \\
\hline Silva $^{19}$ & 2013 & La Araucanía & 87 & RPC muestra orina y endocervix & 15-64 años & No & $\begin{array}{l}11,4 \% \text { cervical } \\
18,3 \% \text { orina }\end{array}$ \\
\hline Conejero $^{18}$ & 2013 & RM & 344 & RPC:autotoma vaginal & 18-25 años & No & $7,9 \%$ \\
\hline Ovalle 21 & 2012 & RM & 255 & RPC muestra cervical & Mujeres embarazadas & Sí & $5,9 \%$ \\
\hline Huneeus $^{16}$ & 2009 & $\mathrm{RM}$ & 203 & RPC: muestra orina & $<25$ años & Sí & $\begin{array}{c}\quad 6,9 \% \text { general } \\
19 \% \text { en mujeres embarazadas }\end{array}$ \\
\hline Martínez ${ }^{17}$ & 2008 & $\mathrm{RM}$ & 403 & RPC: muestra cervical & Mujeres 15 a > 45 & No & $\begin{array}{c}4,7 \% \text { general } \\
8,3 \% \text { en }<25 \text { años }\end{array}$ \\
\hline
\end{tabular}

excluyendo aquellas pacientes que recibieron tratamiento antimicrobiano dentro del mes previo al ingreso al estudio o que estuviesen cursando una enfermedad inflamatoria pélvica. Se tomaron muestras endocervicales y fueron evaluadas por el test de Cobas CT/NG v $2.0\left(\right.$ Roche $\left.^{\circledR}\right)$ el que permite la detección de C. trachomatis y N. gonorrhoeae por RPC. Además, las primeras 100 muestras fueron analizadas con tres métodos diagnósticos. Sólo ocho pacientes estaban embarazadas (4,4\%). Entre los resultados obtenidos destaca que $84,6 \%$ de las pacientes eran de nivel socioeconómico alto, similar al estudio mencionado anteriormente y $85,6 \%$ usaba método de planificación familiar, primando los anticonceptivos orales $(57,5 \%)$ y preservativo $(21,5 \%)$. Además, se describe una diferencia en el número de parejas sexuales entre las que obtuvieron resultado positivo y negativo de 4,1 vs. 2,5 , respectivamente $(\mathrm{p}<0,05)$. Reportaron una prevalencia global de $5,5 \%$, sin especificar la prevalencia en mujeres embarazadas. El análisis de las 100 primeras muestras por los tres métodos moleculares Cobas CT/ NG v2.0 $\left(\right.$ Roche $\left.^{\circledR}\right)$, Cepheid/Xpert CT/NG ${ }^{\circledR}$ (GeneXpert Systems) y Aptima Combo $2^{\circledR}$ mostró $100 \%$ de concordancia (Tabla 1).

Otros estudios previos en poblaciones similares de la Región Metropolitana mostraban resultados semejantes; Martínez y cols., (2008) obtuvieron una prevalencia de $4,7 \%$ siendo mayor en pacientes entre 15 y 26 años (7,3$8,3 \%)^{17}$, mientras que Conejero y cols., refirieron una prevalencia de $7,9 \%{ }^{18}$ (Tabla 1 ).

En la Región de La Araucanía, Silva y cols. (2013) ${ }^{19}$, evaluaron la frecuencia de $C$. trachomatis utilizando $\mathrm{RPC}$ en tiempo real, comparando muestras de orina por auto-toma y muestra endocervical obtenida por un ginecólogo mediante citobrush. Incluyeron 87 mujeres asintomáticas entre 15 y 64 años de dos establecimientos públicos, que acudían a control ginecológico de rutina. Entre los resultados reportaron una frecuencia de 11,4\% en muestra endocervical y $18,3 \%$ en muestra de orina. El $50 \%$ de los casos positivos era menor de 35 años y sólo $4,6 \%$ de los casos tuvo ambas muestras positivas, lo que sugiere que las muestras son complementarias especialmente en pacientes asintomáticas dada la capacidad de C. trachomatis de infectar el tracto genial, la vía urinaria o ambos. Este estudio destaca una de las mayores prevalencias de infección por $C$. trachomatis en población de mujeres no embarazadas reportada en el país (Tabla 1).

En el estudio local más reciente, en la Región de La Araucanía, realizado por Riquelme, Brebi y cols., del Laboratorio de Biología Integrativa de la Universidad de La Frontera, aún no publicado, analizaron la presencia de siete agentes de infecciones de transmisión sexual (ITS) de manera simultánea (C. trachomatis, U. urealyticum, M. hominis y $M$. genitalium, VPH, VHS-1 y VHS-2) en 804 individuos por medio de muestras de orina autorrecolectadas y muestras endocervicales tomadas por citobrush en pacientes asintomáticos y sexualmente activos. De ellos, 72,5\% fueron mujeres, sin especificar si estaban embarazadas. Las muestras fueron analizadas por medio de RPC. Entre los resultados presentan una prevalencia de C. trachomatis de 8,6\%. Esta infección mostró una asociación con pacientes jóvenes entre 18 y 24 años, de medianos ingresos, quienes practican sexo anal, aquellos con dos o más parejas sexuales los últimos tres años y relaciones homosexuales (Tabla 1). 


\section{Infección por Chlamydia trachomatis en el embarazo}

Entre los estudios realizados exclusivamente en mujeres embarazadas, Palma y cols., $(2019)^{20}$ evaluaron la frecuencia de infecciones cervicales en gestantes. Se incluyeron 85 mujeres, de ellas 47 asistían a su control de rutina, sin ITS asociada, y 38 fueron derivadas con ITS asociada (principalmente condilomas acuminados y molusco). Se desarrolló en centros públicos y se excluyeron las pacientes inmunocomprometidas, aquellas con cervicitis clínica, uso antimicrobiano 30 días previos y aquellas con abstinencia sexual menor a tres días. Las muestras fueron recogidas por el profesional y se realizó examen microscópico, cultivos y RPC para N. gonorrhoeae, Trichomonas vaginalis y C. trachomatis. De los resultados reportados destaca que 12,9\% resultaron positivas para Chlamydia y no se encontró N. gonorrhoeae. Del análisis demográfico, sólo 48\% eran chilenas, ya que el resto provenía de otros países latinoamericanos; $16,7 \%$ de las chilenas fueron positivas

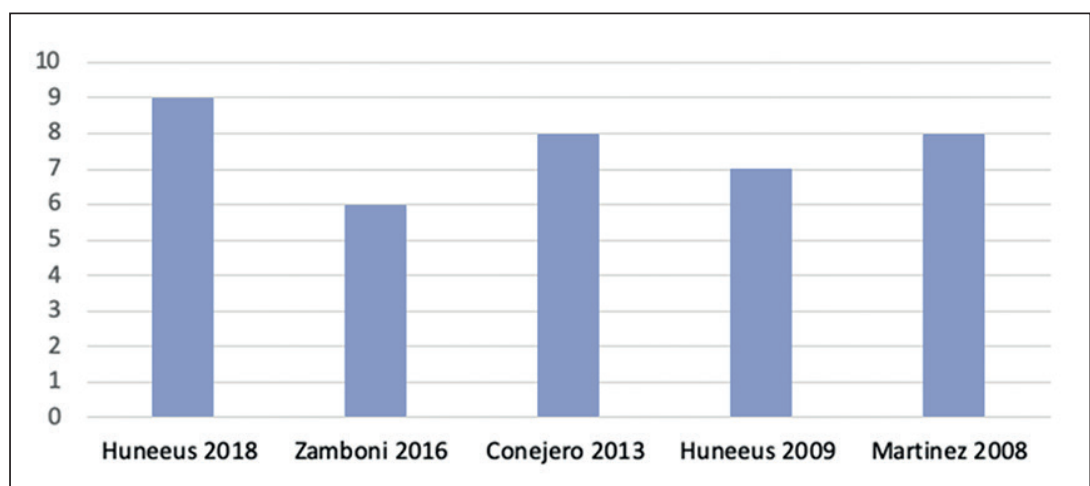

Figura 1. Prevalencia (\%) de C. trachomatis en mujeres menor o igual a 25 años, en estudios chilenos.

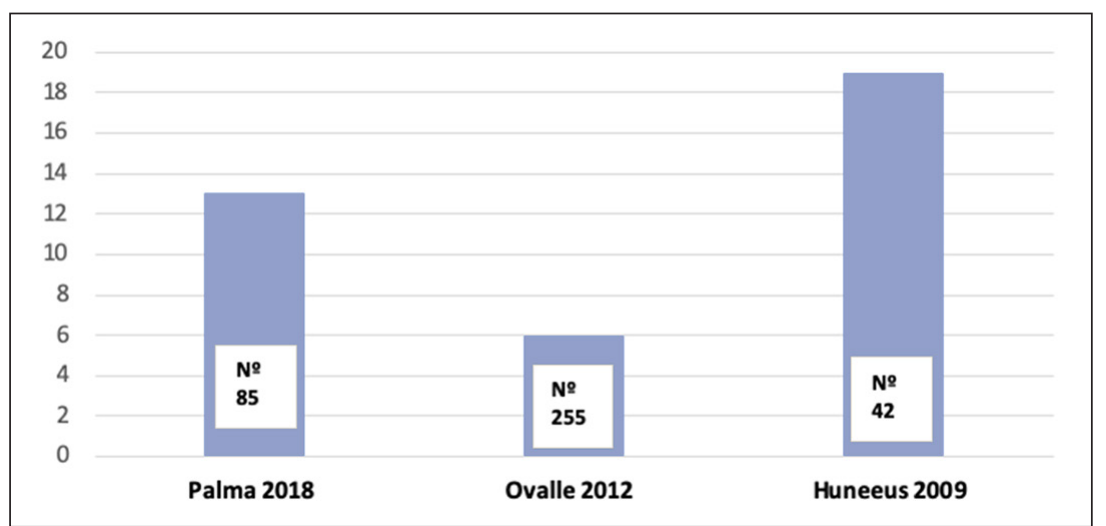

Figura 2. Prevalencia (\%) de C. trachomatis en mujeres embarazadas, en estudios chilenos. frente a $2,6 \%$ de las extranjeras. Por otra parte, $28 \%$ de los casos positivos eran adolescentes entre 16 y 20 años.

Los estudios nacionales, resumidos en la Tabla 1 , muestran una alta prevalencia de $C$. trachomatis en la población femenina de nuestro país, especialmente en población joven y mujeres embarazadas ${ }^{5,15-18}$. La Figura 1 resume la prevalencia de $C$. trachomatis reportada en los estudios chilenos antes descritos, pero en el subgrupo de mujeres menores o iguales a 25 años.

En un estudio reciente de Palma y cols., del año 2018, la prevalencia en mujeres gestantes $(12,9 \%)^{20}$ es mucho mayor respecto de un estudio realizado por Ovalle y cols., seis años antes, quienes analizaron muestras endocervicales de 258 mujeres embarazadas y reportaron una prevalencia de $5,9 \%{ }^{21}$.

La Figura 2 muestra la prevalencia en mujeres embarazadas de ambos estudios y del subgrupo de mujeres embarazadas incluidas en el estudio de Hunneus en $2009^{1}$.

\section{Complicaciones perinatales}

Los recién nacidos pueden infectarse por $C$. trachomatis exponiéndose durante el parto de una madre infectada ${ }^{22}$. La colonización vaginal o rectal del recién nacido puede acercarse a $14 \%$. En estudios con recién nacidos exclusivamente sintomáticos, hijos de madres positivas, el riesgo de desarrollar conjuntivitis fluctúa alrededor de 30 a $40 \%$ y de neumonía, entre 5 y $30 \%$, 23,24 .

El riesgo de adquirir la infección por parte del recién nacido se produce principalmente después de partos vaginales; sin embargo, también se ha reportado luego de cesárea con antecedentes de rotura prematura de membranas, o incluso, aunque menos frecuente, en cesárea con membranas íntegras ${ }^{25,26}$. Bell $\mathrm{T}$ y cols. ${ }^{22}$, publicaron en 1994 un estudio realizado en un hospital universitario que realizaba cribado en mujeres embarazadas. Se estudió a 141 binomios de madres con infección por C. trachomatis, la mayoría de ellas detectadas por dicho cribado. Los recién nacidos fueron estudiados con muestras de conjuntivas, nasofaringe, orofaringe, recto y vagina. Resultaron positivos 67 de 125 nacidos por vía vaginal, 2 de 10 que nacieron por cesárea con membranas rotas y sólo uno de seis nacidos por cesárea con membranas íntegras; en este niño se aisló clamidia de la nasofaringe. Los autores no describen en qué contexto clínico fue esa cesárea ni edad gestacional ni resultado perinatal, pero sugieren la posibilidad de infección transamniótica ascendente.

La manifestación clínica más frecuente en los niños infectados es la conjuntivitis, con un período de incubación de 5 a 14 días. Excepcionalmente, se ha descrito más precoz en casos de rotura prematura de membranas ${ }^{4}$, apoyando la posibilidad de infección ascendente anteparto. Este compromiso ocular fue descrito por Linder y cols. en 
antes de las 20 semanas, y su tratamiento, con la idea de reducir la incidencia de parto prematuro, encontró un solo estudio randomizado, controlado. Incluyó a 4.100 mujeres embarazadas, de ellas, 2.058 cribadas recibieron tratamientos para los microorganismos aislados, y 2.097 (grupo control) sólo pesquisa, con resultado ciego para el equipo tratante, y control obstétrico estándar. El grupo intervenido presentó una tasa de prematuridad significativamente menor que la del grupo control (OR: 0,55; IC $0,41-0,75)$. El riesgo de tener un recién nacido de menos de $1.500 \mathrm{~g}$ fue significativamente más bajo también en el grupo intervenido (RR: 0,34; IC 0,15-0,75). La reducción de prematuros, principalmente de los de muy bajo peso, haría costo/efectiva la realización de dichos programas. La calidad de evidencia fue calificada de moderada por los revisores.

Los estudios que evalúan a un solo agente infeccioso en particular resultan más difíciles de interpretar, dada la naturaleza polimicrobiana de la mayoría de las infecciones intra-amnióticas por una parte $\mathrm{e}^{35}$, y porque diversos agentes de transmisión sexual comparten los mecanismos de adquisición y riesgos de reinfección, haciendo difícil de aislar el impacto de algún microorganismo no estudiado cuando se selecciona sólo uno de ellos para análisis. En este sentido, el mismo grupo de Cochrane, en 2016 hace una revisión sistemática específicamente para C. trachomatis ${ }^{10}$ y su impacto en diversos aspectos de la salud reproductiva. Encontró cuatro estudios aleatorizados, controlados, que incluyeron 21.686 participantes, y mostraron reducción significativa de procesos inflamatorios pelvianos cuando se realizó cribado y tratamiento (RR 0,68; IC: 0,49-0,94). La misma revisión sistemática no encontró estudios de calidad adecuada para análisis en población de mujeres embarazadas.

Una revisión sistemática efectuada en 2019 por Kanninen y cols. ${ }^{39}$, evaluó la prevalencia de C. trachomatis en mujeres embarazadas que ingresaron por amenaza de parto prematuro comparada con mujeres gestantes controles sin amenaza. El estudio infeccioso se realizaba con RPC o cultivo. Incluyó cuatro estudios de cohorte o caso-control, con 591 mujeres embarazadas. La prevalencia de infección por C. trachomatis fue significativamente mayor en las mujeres con amenaza de parto prematuro. E1 9\% de dichas pacientes eran positivas, en comparación al 1\% de las pertenecientes al grupo control (OR: 7,74; IC: 2,64-22,7), por lo que los autores sugirieron incorporar el estudio de este agente en las pacientes con amenaza de parto prematuro. Otra revisión sistemática, de Ahmadi A y cols., publicada en $2018^{36}$, incluyó 23 estudios que analizaban la relación entre la infección por este agente y parto prematuro. Incluían poblaciones de Europa, Asia, África y E.U.A. El metaanálisis mostró que las mujeres embarazadas infectadas tenían un riesgo significativamente mayor de parto prematuro (OR: 2,16; IC: 1,3-3,5). 
Muy interesante resulta analizar el estudio de cohorte poblacional de Reekie J y cols., publicado también en 2018, y las discusiones científicas que ha motivado posteriormente $\mathrm{e}^{40,-42}$. Este trabajo de Western Australia, utilizó el registro de mujeres en edad reproductiva (nacidas entre 1974 y 1995), el registro de partos entre 2001 y 2012, y el registro del programa de cribado existente en ese mismo período. Así, 101.558 mujeres tuvieron un parto único, con 3,9\% de parto prematuro espontáneo. 21.267 mujeres fueron estudiadas durante el embarazo, con 6,4\% de positividad. 19.157 mujeres tenían cribado previo al embarazo, con $8,3 \%$ de resultados positivos. El estudio reporta que las mujeres gestantes que fueron testeadas antes del embarazo presentaban un aumento de riesgo de parto prematuro, no así las testeadas durante la gestación. Entre las que tuvieron test positivo durante el embarazo, $6,2 \%$ tuvo un parto prematuro espontáneo, mientras que esto ocurrió en 4,5\% de las negativas. El análisis multivariado no mostró diferencias significativas entre estas cifras. Tampoco hubo diferencias en riesgo de mortinato. Los autores, por el modelo de estudio, desconocían la existencia o no de tratamiento luego del test, elemento que resulta clave en la interpretación de los resultados. Sin embargo, basados en auditorías clínicas previas en la misma población, ellos asumieron en su discusión que la mayoría fue tratada cuando el cribado resultó positivo para C. trachomatis.

Teniendo eso en consideración, el realizar cribado intraembarazo (y tratar a las positivas) permitiría a este grupo de mujeres embarazadas tener una tasa de prematuridad y de muerte fetal similar al del grupo de gestantes que resultaban negativas en el cribado, reforzando la importancia de dicha intervención.

Ese beneficio no se observó en quienes recibieron tamizaje sólo antes del embarazo. Si las parejas no son adecuadamente tratadas y/o si la paciente persiste en conducta de riesgo, cualquier intervención propuesta en la mujer embarazada podrá sufrir de una reducción en su beneficio ${ }^{43}$, ya que se sabe que 10 a $15 \%$ de las personas positivas tratadas, estarán re-infectadas a los tres meses. Ésta podría ser una de las explicaciones de por qué los autores encontraron un aumento del riesgo de prematuridad entre las testeadas antes de la gestación, cuando se les compara con las mujeres embarazadas con cribado intraembarazo negativo y con las que resultaron positivas en la gestación y probablemente recibieron tratamiento.

\section{Cribado en el embarazo}

Las políticas de cribado prenatal varían alrededor del mundo. Al 2016 sólo 13 países contaban con estos programas, en Europa, Asia, Norteamérica y Oceanía ${ }^{44}$. En
América latina no existen países que hayan incorporado esta política aún.

Por ejemplo, el USPSTF recomienda desde el año $2007^{45}$ hacer tamizaje todos los años a todas las mujeres sexualmente activas no embarazadas de menos de 25 años de edad, como una recomendación A; y realizar cribado a las mujeres embarazadas de menos de 25 años, como una recomendación B. El CDC de E.U.A. recomienda el tamizaje periódico en mujeres sexualmente activas desde 1993, y posteriormente incorporó también la recomendación de hacerlo en mujeres embarazadas. Ellos recomiendan el cribado en el primer control prenatal en todas las mujeres embarazadas menores de 25 años o mayores si tienen algún factor de riesgo como múltiples parejas sexuales, pareja sexual con parejas intercurrentes o con alguna ITS. Luego recomiendan volver a testear en el tercer trimestre si son menores de 25 años o mayores que persistan con los factores de riesgo ${ }^{8,46,47}$. La Public Health Agency, de Canadá, sugiere control anual en mujeres de menos de 25 años $\mathrm{y}$ en el primer control prenatal de todas las mujeres embarazadas con factores de riesgo ${ }^{48}$. El Government of Western Australia también propone el tamizaje en control ginecológico y en el primer control prenatal de las menores de 30 años ${ }^{49}$.

En cambio, el National Health System (NHS) en el Reino Unido, tiene un programa nacional de pesquisa de $C$. trachomatis, en el que testean a todos los jóvenes entre 15 y 24 años sexualmente activos, con periodicidad anual o cuando cambian de pareja sexual ${ }^{50}$. Hasta el momento, desaconsejan realizar testeo en mujeres embarazadas como parte del control prenatal. Su última actualización la realizaron en $2018^{43,51}$ e igualmente recomiendan que, si la gestante es menor a 25 años y tiene factores de riesgo, debe ser derivada al programa nacional de cribado ${ }^{52}$.

En 2015, Ong J y cols., realizaron un estudio de costo-efectividad para incorporar cribado a las mujeres embarazadas de menos de 25 años como parte del control prenatal de Australia. Ellos mostraron que con una prevalencia de $3 \%$ de infección por C. trachomatis, el incorporar cribado en ese grupo resulta costo-efectivo ${ }^{53}$. Y Rours G y cols. ${ }^{8}$, en 2016, evaluaron esto para la población de Países Bajos, que tenía descrita una prevalencia de $3,9 \%$; ellos encuentran que el cribado es costo-efectivo si se realiza a todas las mujeres embarazadas, y más aún si se selecciona a las menores de 30 años.

\section{Cribado en el embarazo, situación en Chile}

En nuestro país no existen políticas públicas de cribado en grupos de riesgo ni en mujeres embarazadas. Recién durante el año 2019 se incorporó la codificación en FO- 
gran oportunidad para adherir a un programa de cribado ( 90 vs. $37 \%)^{8}$ y adherir a realizar el tratamiento en caso de resultar positiva. En cambio, las mujeres de menos de 25 años no embarazadas acuden en forma más infrecuente, esporádica e irregular a controles de salud reproductiva o sexual. Se debe tener en consideración que, por ejemplo, el protocolo de prevención de cáncer cérvico-uterino comienza recién a partir de los 25 años.

- El cribado y el adecuado tratamiento de la paciente y su pareja disminuye la prevalencia en el grupo intervenido, e indirectamente protege a futuras parejas sexuales $^{8}$

- El cribado permite otorgar una buena oportunidad de educación en prevención de infecciones de transmisión sexual, y reforzarlo durante el resto del control prenatal.

- Existe evidencia indirecta, expuesta acá, de que la pesquisa y tratamiento de la infección por este agente podría reducir el riesgo de parto prematuro.

- La prematuridad sigue siendo en nuestro país una de las principales causas de mortalidad perinatal y de secuelas neurológicas originadas en el período perinatal, y la reducción de su incidencia sólo se logra a través de la suma de intervenciones diferentes dada su naturaleza multicausal.

\section{Comentario final}

La infección genital por C. trachomatis corresponde a la enfermedad de transmisión sexual bacteriana más frecuente en el mundo. Tiene un enorme impacto en la salud sexual y reproductiva, principalmente en relación a proceso inflamatorio pelviano y su secuela de infertilidad y embarazo ectópico, pero también hacia el recién nacido, ya sea por la infección directa en el parto, como probablemente también a través del riesgo de prematuridad y sus secuelas.

La prevalencia de esta infección en nuestro país, y en nuestra región, es elevada, y similar a lo descrito en la literatura internacional. En ese contexto, existe un amplio consenso de instituciones internacionales recomendando el cribado de mujeres en grupos etarios de mayor riesgo, y algunas de ellas, también lo recomiendan en el embarazo.

Chile presenta un aumento de las ITS, enfermedades que son de notificación obligatoria, pero se desconoce el impacto de C. trachomatis, al no pertenecer a ellas. Creemos que es el momento de incorporar su estudio, tratamiento precoz y vigilancia de reinfecciones, en forma sistemática a nivel nacional. La mujer embarazada, al menos de grupo etario de mayor riesgo o con otra ITS, y la que tenga riesgo de parto prematuro debiese estar considerada también en dicho Plan Nacional de Cribado. 


\section{Referencias bibliográficas}

1.- World Health Organization. Guidelines for the treatment of Chlamydia trachomatis. [Internet] Suiza: World Health Organization; 2016 [Citado el 10 de junio de 2020]. Disponible en: https://apps.who.int/iris/bitstream/hand le/10665/246165/9789241549714-eng.pdf;jsess ionid=BD28F9646988F52482A01238079554F 7 ? sequence $=1$.

2.- Centers for Disease Control and Prevention. Reported STDs in the United States, 2018 [Internet] Centers for Disease Control and Prevention; 2019. [Citado el 10 de junio de 2020] Disponible en: https://www.cdc.gov/ nchhstp/newsroom/docs/factsheets/STDTrends-508.pdf.

3.- Centers for Disease Control and Prevention. Sexually Transmitted Disease Surveillance, 2018: Women and infants [Internet] Centers for Disease Control and Prevention; 2019. [Actualizado el 30 de Julio del 2019; citado el 13 de junio de 2020]. Disponible en: https:// www.cdc.gov/std/stats18/womenandinf.htm.

4.- Hammerschlag M. Chlamydia trachomatis infections in the newborn. [Internet] UpToDate, 2018. [Actualizado 2020; citado el 10 de octubre de 2020] Disponible en: https://www. uptodate.com/contents/chlamydia-trachomatisinfections-in-the-newborn\#H24.

5.- Zamboni M, Ralph C, García P, Cuello M. La prevalencia actual de infección genital por Chlamydia trachomatis en adolescentes y mujeres jóvenes chilenas asintomáticas justifica la vigilancia periódica. Rev Chilena Infectol. 2016; 33(6): 619-27. doi: 10.4067/S071610182016000600003

6.- Stamm W. Capítulo 7: Enfermedades infecciosas, Sección 10: Infecciones por Chlamydia. En: Fauci A, editor. Harrison Principios de Medicina Interna, Volumen 1, $17^{\circ}$ ed. México: Mc Graw Hill; 2009. p.1080-1070.

7.- Jennings L K, Krywko D M. Pelvic Inflammatory Disease. [Internet] Isla Treasure: StatPearls Publishing; 2020 [Actualizado el 20 de noviembre del 2020; citado el 10 de Junio de 2020]. Disponible en: https://www.ncbi.nlm. nih.gov/books/NBK499959/

8.- Rours G I, Smith-Norowitz T A, Ditkowsky J, Hammerschlag M R, Verkooyen R $\mathrm{P}$, de Groot R, et al. Cost- effectiveness analysis of Chlamydia trachomatis screening in Dutch pregnant women. Pathog Glob Health. 2016; 110:(7-8): 292-302. doi: 10.1080/20477724.2016.1258162.

9.- Martínez M A, Millán F, González C. Chlamydia trachomatis genotypes associated with pneumonia in Chilean infants. Scand J Infect Dis 2009; 41: 313-6. doi: 10.1080/00365540902744758.

10.- Low N, Redmond S, Uusküla A, van Bergen J, Ward H, Andersen B, et al. Screening for genital chlamydia infection. Cochrane Database Syst Rev 2016; (9). doi: 10.1002/14651858. CD010866.pub2.

11.- Low N. Sex, Chlamydia trachomatis and reproductive health: what can we learn from systematic reviews of observational studies? Sex Transm Infect, 2020; 96(5): 315-7. doi: 10.1136/sextrans-2019-054279.

12.- Viscarra T, Brebi P, Andana A, Sánchez R. Infecciones de transmisión sexual en semen. El hombre como vector de transmisión. Int. J. Morphol. 2013; 31(1): 254-63. doi: http://dx.doi.org/10.4067/S071795022013000100041.

13.- Retamal J, Sánchez R, Brebi P. Infecciones de transmisión sexual silentes: la muestra de orina permite una adecuada detección. Rev Chilena Infectol 2015; 32 (3): 283-8. doi: http://dx.doi org/10.4067/S0716-10182015000400005.

14.- Levy SB, Gunta J, Edemekong P. Screening for sexually transmitted diseases. Prim Care 2019; 46: 157-73. doi: 10.1016/j.pop.2018.10.013

15.- Huneeus A, Schilling A, Fernández M I. Prevalence of Chlamydia trachomatis, Neisseria gonorrhoeae, and Trichomonas vaginalis infection in chilean adolescents and young adults. J Pediatr Adolesc Gynecol, 2018; 31(4): 411-5. doi: 10.1016/j.jpag.2018.01.003.

16.- Huneeus A, Pumarino M G, Schilling A, Robledo P, Bofil M. Prevalencia de Chlamydia trachomatis y Neisseria gonorrhoeae en adolescentes chilenas. Rev Med Chile 2009; 137(12): 1569-74. doi: http://dx.doi. org/10.4067/S0034-98872009001200004.

17.- Martínez M A, Reid I, Arias C, Napolitano C, Sandoval J, Molina R. Prevalencia de infección cervical por Chlamydia trachomatis en mujeres de la Región Metropolitana. Rev Med Chile 2008; 136: 1294-300. doi: http://dx.doi.org/10.4067/ S0034-98872008001000009.

18.- Conejero C, Cannoni G, Merino P, Bollmann J, Hidalgo C, Castro M, et al. Experiencia con un método de autotoma de muestra vaginal para la detección de infección por Chlamydia trachomatis y Neisseria gonorrhoeae en mujeres jóvenes. Rev Chilena Infectol 2013; 30(5): 489-93. doi: http://dx.doi.org/10.4067/ S0716-10182013000500004.

19.- Silva R, León D, Viscarra T, Ili C, Roa J C, Sánchez R, et al. Frecuencia de la infección por Chlamydia trachomatis en un grupo de mujeres de la Región de la Araucanía, Chile. Rev Chilena Infectol 2013; 30(6): 611-5. doi: http://dx.doi.org/10.4067/S071610182013000600006.

20.- Palma C, Martínez M A, Santander E. Cribado de infecciones cervicales de transmisión sexual en embarazadas y su relación con la microbiota vaginal. Rev Chilena Infectol 2019; 36(3): 292-8. doi: http://dx.doi.org/10.4067/S071610182019000300292.
21.- Ovalle A, Martínez M A, de la Fuente F, Falcon N, Feliú F, Fuentealba F, et al. Prevalencia de infecciones de transmisión sexual en mujeres embarazadas atendidas en un hospital público de Chile. Rev Chilena Infectol 2012; 29(5): 517-20. doi: http://dx.doi. org/10.4067/S0716-10182012000600006.

22.- Bell T, Stamm W, Kuo C, Wang S, Holmes $\mathrm{K}$, Grayston J. Risk of perinatal transmission of Chlamydia trachomatis by mode of delivery. Journal of Infection, 1994; 29 (2):165-9. doi: https://doi.org/10.1016/S01634453(94)90674-2.

23.- Heggie A D, Lumicao G G, Stuart L A, Gyves M T. Chlamydia trachomatis infection in mothers and infants. A prospective study. Am J Dis Child. 1981; 135 (6): 507-11. doi:10.1001/ archpedi.1981.02130300007005.

24.- Rosenman M B, Mahon B E, Downs S M, Kleiman M B. Oral erythromycin prophylaxis vs watchful waiting in caring for newborns exposed to Chlamydia trachomatis. Arch Pediatr Adolesc Med. 2003; 157(6): 565-71. doi: 10.1001/archpedi.157.6.565.

25.- Shariat H, Young M, Abedin M. An interesting case presentation: a possible new route for perinatal acquisition of Chlamydia. J Perinatol. 1992; 12(3): 300-2. PMID: 1432291.

26.- Bell T A. Chlamydia trachomatis infection in dizygotic twins delivered by caesarean section. Genitourin Med 1988; 64(5): 347-8. doi: 10.1136/sti.64.5.347.

27.- Lindner K. Gonoblennorrhoe, einschlussblennorrhoe und trachom. Albrecht von Graefe's Arch Ophthalmol. 1911; 78: 34580 .

28.- Darville T. Chlamydia trachomatis infections in neonates and young children. Semin Pediatr Infect Dis. 2005; 16(4): 235-44. doi: 10.1053/j. spid.2005.06.004.

29.- Jones B R, Al-Hussaini M K, Dunlop E M. Genital infection in association with TRIC virus infection of the eye: I. Isolation of virus from urethra, cervix, and eye: preliminary report. $\mathrm{Br}$ J Vener Dis. 1964; 40(1): 19-24. doi: 10.1136/ sti.40.1.19

30.- Beem M, Saxon E. Respiratory tract colonization and a distinctive pneumonia syndrome in infants infected with Chlamydia trachomatis. N Eng J Med, 1977; 296: 306-10. doi: 10.1056/NEJM197702102960604.

31.- Hammerschlag M. Chlamydial and gonococcal infections in infants and children. Clin Infect Dis. 2011; 53 (3): 99-102. doi: 10.1093/cid/ cir699

32.- Shinkwin C A, Gibbin K P. Neonatal upper airway obstruction caused by chlamydial rhinitis. J Laryngol Otol 1995; 109(1): 58-60. doi: $10.1017 / \mathrm{s} 002221510012924 x$.

33.- Workowski K A, Bolan G A, Centers for Disease Control and Prevention. Sexually transmitted diseases treatment guidelines, 2015. 
MMWR Recomm Rep 2015; 64: 1-137. https:// pubmed.ncbi.nlm.nih.gov/26042815/

34.- LeFevre M L, U.S. Preventive Services Task Force. Screening for Chlamydia and gonorrhea: U.S. Preventive Services Task Force recommendation statement. Ann Intern Med 2014; 161(12): 902-10. doi: 10.7326/M141981.

35.- Kim C J, Romero R, Chaemsaithong $P$, Chaiyasit N, Yoon B H, Kim Y M. Acute chorioamnionitis and funisitis: definition, pathologic features, and clinical significance. Am J Obstet Gynecol. 2015; 213(4): S29-52. doi: 10.1016/j.ajog.2015.08.040.

36.- Ahmadi A, Ramazanzadeh R, Sayehmiri K, Sayehmiri F, Amirmozafari N. Association of Chlamydia trachomatis infections with preterm delivery; a systematic review and metaanalysis. BMC Pregnancy and Childbirth. 2018; 18: 240. doi: https://doi.org/10.1186/s12884018-1868-0

37.- Olson-Chen C, Balaram K, Hackney D N. Chlamydia trachomatis and adverse pregnancy outcomes: meta-analysis of patients with and without infection. Matern Child Health J. 2018; 22(6): 812-21. doi: 10.1007/s10995-0182451-z.

38.- Sangkomkamhang U S, Lumbiganon P, Prasertcharoensuk W, Laopaiboon M. Antenatal lower genital tract infection screening and treatment programs for preventing preterm delivery. Cochrane Database Syst Rev 2015;(2) doi: 10.1002/14651858.CD006178.pub3

39.- Kanninen T T, Quist-Nelson J, Sisti G, Berghella V. Chlamydia trachomatis screening in preterm labor: A systematic review and meta-analysis. Eur J Obstet Gynecol Reprod Biol. 2019; 240: 242-7. doi: 10.1016/j. ejogrb.2019.06.032.

40.- Reekie J, Roberts C, Preen D, Hocking J S, Donovan B, Ward J, et al. Chlamydia and Reproductive Health Outcome Investigators. Chlamydia trachomatis and the risk of spontaneous preterm birth, babies who are born small for gestational age, and stillbirth: a population-based cohort study. Lancet Infect Dis. 2018; 18(4): 452-60. doi: 10.1016/S14733099(18)30045-8.

41.- Adamson P C, Klausner J D. Treating chlamydial infections in pregnancy and preventing adverse birth outcomes. Lancet Infect Dis. 2018; 18(4): 368-9. doi: 10.1016/ S1473-3099(18)30049-5.
42.- Xiang-Sheng Chen. Adverse pregnancy outcomes due to Chlamydia trachomatis. Lancet Infect Dis. 2018. 18 (5); 499. doi: https://doi.org/10.1016/S14733099(18)30211-1.

43.- York Health Economics Consortium: Fitzgerald A, Cikalo M, Isojarvi J, Shaw A, Edwards M, Glanville J. Chlamydia Screening in pregnancy: external review against programme appraisal criteria for the UK National Screening Committee. [Internet] Londres, 13 de marzo de 2018. [Citado el 13 de junio de 2020]. Disponible en: https:// legacyscreening.phe.org.uk/policydb_ download.php?doc $=1003$

44.- Davey J, Medline A, Klausner J D. Screening pregnant women in the 2015 European guideline on the management of Chlamydia trachomatis infections. Int J STD AIDS

45.- U.S. Preventive Services Task Force. Screening for chlamydial infection: U.S. Preventive Services Task Force recommendation statement. Ann Intern Med. 2007; 147(2): 12834. doi: 10.7326/0003-4819-147-2-20070717000172 .

46.- Centers for Disease Control. Sexually Transmitted Disease: Detailed Fact Sheet. [Internet] Centers for Disease Control; 2016. [Citado el 18 de mayo de 2020] Disponible en: https://www.cdc.gov/std/pregnancy/stdfactpregnancy-detailed.htm.

47.- Hoover K, Leichliter J, Torrone E, Loosier P, Gift T, Tao G; Centers for Disease Control and Prevention (CDC). Chlamydia screening among females aged 15-21 years--multiple data sources, United States, 1999-2010. MMWR Suppl. 2014; 63(2): 80-8. https://pubmed.ncbi. nlm.nih.gov/25208262/

48.- Government of Canada. Canadian Guidelines on Sexually Transmitted Infections - Specific populations - Pregnancy [Internet] Government of Canada; 2017. [Actualizado el 31 de agosto de 2017; citado el 18 de mayo de 2020]. Disponible en: https:// www.canada.ca/en/public-health/services/ infectious-diseases/sexual-health-sexuallytransmitted-infections/canadian-guidelines/ sexually-transmitted-infections/canadianguidelines-sexually-transmitted-infections- 41 $\mathrm{html}$.

49.- Microbiology Consultant PathWest. Sexually transmitted infections: Chlamydia in pregnancy [Internet] Goverment of Western
Australia North Metropolitan Health Service Women and Newborn Health Service; 2008 [Actualizado en marzo del 2020; citado el 18 de mayo de 2020] Disponible en: https:// www.wnhs.health.wa.gov.au/ /media/ Files/Hospitals/WNHS/For\%20health\%20 professionals/Clinical\%20guidelines/OG/ WNHS.OG.SexuallyTransmittedInfectionsChlamydiainPregnancy.pdf.

50.- Public Health England. Opportunistic chlamydia screening of young adults in england an evidence summary [Internet] Londres: Public Health England; 2014. [citado el 18 de mayo de 2020] Disponible en: https://assets. publishing.service.gov.uk/government/uploads/ system/uploads/attachment data/file/740182/ Opportunistic_Chlamydia_Screening Evidence Summary April 2014.pdf.

51.- UK National Screening Committee. The UK NSC recommendation on Chlamydia screening in pregnancy. [Internet] UK National Screening Committee; abril 2018. [citado el 18 de mayo de 2020] Disponible en: https:// legacyscreening.phe.org.uk/chlamydiapregnancy.

52.- National Collaborating Centre for Women's and Children's Health. Antenatal care: routine care for the healthy pregnant woman. [Internet] Londres: National Collaborating Centre for Women's and Children's Health 2008. [citado el 18 de mayo de 2020] 184-193 p. Disponible en: https://www.nice.org.uk/guidance/cg62/ evidence/evidence-tables-from-the-2003version-pdf-196748322.

53.- Ong J, Chen M, Hocking J, Fairley C K, Carter R, Bulfone L, Hsueh A. Chlamydia screening for pregnant women aged 16-25 years attending an antenatal service: a cost-effectiveness study. BJOG. 2016; 123(7): 1194-202. doi: 10.1111/1471-0528.13567.

54.- Huneeus A, Soriano H, Pommer R, Delpiano L, Salas F, Céspedes P, et al. Documento: Chlamydia trachomatis: fundamentos de la importancia del cribado en el sistema público de salud. Rev Chilena Infectol 2018; 35(5): 498-500. doi: http://dx.doi.org/10.4067/s071610182018000500498

55.- Cáceres K. Departamento Epidemiología MINSAL. Informe: Situación epidemiológica de las infecciones de transmisión sexual en Chile, 2017. Rev Chilena Infectol 2019; 36(2): 221-33, doi: http://dx.doi.org/10.4067/S071610182019000200221 